\title{
Providing Access to Multilingual Patient Education Materials
}

\begin{abstract}
Nicole Askin
Access to reliable yet readable health information is key for consumers making health decisions and participating in the management of their care. This presents a particular challenge for patients whose first language is not English: the language barrier makes it difficult for them to understand the counsel and direction of care providers, educational materials available at clinics are most typically in English, and online reliable sources in their own language may be difficult to locate, particularly for those with additional literacy or technological barriers. This article will highlight a pilot project to collate online patient education materials in languages other than English for use by providers in primary care.
\end{abstract}

Keywords: health literacy, multilingual resources, hospital librarians, patient education

Nicole Askin, MLIS (nicole.askin@umanitoba.ca), Acting Hospital Librarian, Seven Oaks General Hospital Library, University of Manitoba Health Sciences Libraries, 2300 McPhillips St, Winnipeg, Manitoba, Canada, R2V 3M3 


\section{INTRODUCTION}

Medical librarians play an important role in providing educational materials to patients to help them better understand their health and participate in their care. These materials should be responsive to the needs of local patients and clinicians, in terms of both the topics covered and the manner in which they are conveyed. When the author attended a local primary-care centre to present the library's patient education materials to clinicians there, two major questions were raised: what is the reading level of these materials, and in what languages are they available. Means of addressing the first question were explored by Leonard (2017) in a previous issue; the second question led to the development of a pilot project to collate online patient education materials in languages other than English for use by providers in primary care.

\section{BACKGROUND}

The University of Manitoba Health Sciences Libraries (UMHSL) system supports the students and staff of the university as well as staff at the Winnipeg hospitals, health centres and community-based health facilities, among other patron groups. Libraries located at each hospital provide service to a catchment area of the city. Consumer health materials can be borrowed at UMHSL locations with a free Consumer Health card; the collection is supported by the Dr.

Georgina R. Hogg Endowment Fund. Most of the print materials in the collection are in English.

The UMHSL is located in Winnipeg, Manitoba, Canada. The official languages of Canada are English and French, but an estimated 23\% of city residents spoke another mother tongue as of 2011. 24 non-official languages had over 1000 speakers in the city, according to 
census records (Statistics Canada 2012). The Seven Oaks General Hospital Library is located in the northwest area of the city, which has large concentrations of speakers of German, Punjabi, Polish, and Tagalog, and significant concentrations of speakers of a number of additional languages (Statistics Canada 2012). This library serves the Seven Oaks General Hospital and several area primary care clinics, such as the NorWest Co-op Community Health Centre, with large numbers of clients who speak languages other than English. Staff at NorWest requested that the library examine the possibility of providing or recommending resources in the languages spoken by their clients. This request was the impetus for this pilot project.

\section{LITERATURE REVIEW}

Multiple authors have previously examined the problem of patient education for individuals with a first language other than English. Discussion of patients with limited English proficiency fits into a broader discussion of those with low health literacy, or a limited ability to understand health-related information. A systematic review by Berkman and colleagues found that low health literacy was associated with a range of negative health-related outcomes, including increased number of hospitalizations, higher mortality rates, poor compliance with medication instructions, and lower rates of screening and preventative health care. McKee and PaascheOrlow (2012) discussed the potential of applying research around low health literacy to the problem of patient education for non-English speakers. They noted that both populations are likely to experience negative socioeconomic determinants of health, including but not limited to reduced access to information and reduced access to care. Sentell and Braun (2012) found that low health literacy and limited English proficiency frequently co-occurred, but that the latter may 
correlate to greater risk for poor health outcomes. This research motivates efforts to provide patient education materials in the language in which they are most likely to be understood.

Perera and collaborators (2012) investigated whether providing patients with educational materials in their own language would improve their understanding of their condition and treatment. They found that it did, and that patients preferred having these materials in a language they could more easily understand. Workman and others (2003) advocated for libraries to be involved in providing patient education materials in languages other than English, noting that most online content is in English only. They suggest working with care providers that serve nonEnglish-speaking patients to better understand the needs of clients in the local context.

Weiss and colleagues (2007) focused on medication instructions, given that correctly administering medications at home is primarily a patient responsibility. They found that although $90 \%$ of pharmacists surveyed reported serving non-English-speaking clients on a daily basis, there were significant gaps both in identifying those needing assistance and providing linguistically appropriate medication labelling. Bailey and colleagues (2012) sought to address the problems raised by Weiss and others by developing prescription instructions in multiple languages. Their process was first to create instructions aimed at those with low health literacy and then to iteratively translate these into their target languages. They found this process to be cost- and time-intensive, and so potentially unusable for those in resource-limited situations.

Several authors have proposed alternate means of providing access to non-English patient education materials, other than developing them locally. Friedman and colleagues (2005) discussed the creation of a health information site in Russian. The site's contents are largely patient education materials developed by various English-language organizations, which have been collated in a single site to make them easily locatable for those seeking Russian-language 
resources. Plumbaum and others (2014) developed a health information system to allow patients to search for materials in their native language, including a multilingual ontology. Charbonneau and Workman (2002) described a project of digitizing multilingual health education resources to a web portal where they could be searched and downloaded. All of these initiatives take as their basis the problem that it is currently difficult for inexpert searchers to locate reliable health information online, particularly in languages other than English. This is the problem that the pilot project attempts to address.

\section{PROJECT}

As a result of the meeting with NorWest staff, patient education materials in languages other than English and at lower reading levels will be considered for long-term collection development. However, it is not feasible to quickly develop a broad collection in all languages spoken in the community. Thus, alternative, lightly resourced means of providing such materials to clinicians needed to be considered. Bibliographies of recommended resources for various topics already existed in English, so this approach was chosen to highlight equivalent resources in other languages. The project began by identifying a core list of multilingual health websites, and then collating resources specific to each topic and language of interest.

Three types of topics were selected for specific handout development: broad systems-based overviews (eg. heart disease), major regional conditions and priorities (eg. diabetes), and specific topics requested by the NorWest clinicians (eg. smoking cessation). Two sets of languages were prioritized: those with large groups of speakers in the area as identified by census records (eg. Punjabi), and those identified as priority languages by the NorWest clinicians 
(eg. Arabic). Supplemental languages with smaller groups of speakers in the area were included where possible. Handouts were organized by topic and subdivided by language, with one page for each language (see Figure 1 for an example).

\section{[PLACE FIGURE 1 HERE]}

\section{FIGURE 1. Front page of the diabetes handout, showing available languages.}

As with the English-language versions, the handouts were posted on the library website and also made available in hard copy for patrons or clinicians. However, unlike the English versions, the multilingual handouts consist almost entirely of web sources. Thus, to facilitate the use of hard copies, bit.ly was used to create shortened URLs that could more easily be typed into a web browser. Each link was accompanied by 2-3 words that described the topic, to allow clinicians to quickly locate the most relevant resource; for example, the Diabetes handout included entries for foot care and glucose monitoring (see Figure 2).

\section{[PLACE FIGURE 2 HERE]}

\section{FIGURE 2. Diabetes in Cree handout}

As noted by Friedman and colleagues (2005), most of the reliable resources located online were developed by English-language organizations, including governments and nonprofits. For this project Canadian materials were prioritized where possible because of their applicability to the local context and for avoidance of confusion. For example, the New South Wales (Australia) Ministry of Health has a number of excellent resources on their Multicultural Health Communication website (<mhcs.health.nsw.gov.au>), but these advised patients to call 000 in an emergency, rather than the US and Canadian equivalent 911. Some resources provided a side-by-side English translation of the non-English text, which was very helpful in assessing compatibility with local practice. 
Ongoing maintenance and evaluation will be a necessary component of future work on this project. As with any web-based resources, the entries in these handouts are subject to link rot and need to be checked regularly. They are also updated in response to clinician feedback and, when library staff is aware of them, newly published resources. Thus far, there has been no direct feedback from patients with regards to the utility of their care providers or they themselves having access to these handouts. While it is expected based on the work of Perera and colleagues (2012) among others that this patient education opportunity would have a positive impact, direct evaluation of this particular context would be an avenue for further work. In particular, it would be helpful to understand which sources of material are and are not helpful, to better guide further development of the handouts. Evaluations of video versus print-only patient education materials 
have been conducted in English (cf. Laszewski et al 2016; Prakash et al 2013); it would be useful to know whether these findings hold true in the context of non-English-speaking patients.

Another area for future work is the development of print collections in languages other than English. While web resources are convenient in that they can be viewed and printed from anywhere with Internet access, in some contexts print is preferable. The library has added several non-English resources to its collection in response to requests from clinicians. The local public library also has patient education materials in various languages, although as their indexing is incomplete they can be difficult for inexpert searchers to locate and use. Ideally local healthcare organizations could develop context-specific print resources on various health topics, but because of resource limitations this is unlikely in the near future.

\section{REFERENCES}

Bailey, Stacy Cooper, Romana Hasnain-Wynia, Alice Hm Chen, Urmimala Sarkar, Alisu Schoua-Glusberg, Lee A. Lindquist, Dean Schillinger, and Michael S. Wolf. 2012. "Developing Multilingual Prescription Instructions for Patients with Limited English Proficiency.” Journal of Health Care for the Poor and Underserved 23(1): 81-87. doi:10.1353/hpu.2012.0000.

Berkman, Nancy D., Stacey L. Sheridan, Katrina E. Donahue, David J. Halpern, and Karen Crotty. 2011. "Low Health Literacy and Health Outcomes: An Updated Systematic Review." Annals of Internal Medicine 155(2): 97-107. doi:10.7326/0003-4819-155-2-201107190-00005. Charbonneau, Deborah H., and T. Elizabeth Workman. 2002. "Providing Online Health Information in Many Languages." Journal of Hospital Librarianship 2(3): 39-49. doi:10.1300/J186v02n03_04. 
Friedman, Yelena, Luda Dolinsky, and Rimma Perelman. 2005. "Providing Access to Consumer Health Information for the Russian-Speaking Population.” Journal of Consumer Health on the Internet 9(1): 25-32. doi:10.1300/J381v09n01_03.

Laszewski, Pamela, Cynthia Zelko, Lena Andriths, Eva Vera Cruz, Carole Bauer, and Morris A. Magnan. 2016. "Patient Preference for Instructional Reinforcement Regarding Prevention of Radiation Dermatitis." Clinical Journal of Oncology Nursing 20(2): 187-91. doi:10.1188/16.CJON.187-191.

Leonard, Kelsey. 2017. "Evaluating Patient Education Materials for Grade Level.” Journal of Consumer Health on the Internet 21(1): 87-94. doi:10.1080/15398285.2017.1280347.

McKee, Michael M., and Michael K. Paasche-Orlow. 2012. "Health Literacy and the Disenfranchised: The Importance of Collaboration between Limited English Proficiency and Health Literacy Researchers.” Journal of Health Communication 17(Suppl 3): 7-12. doi:10.1080/10810730.2012.712627.

Perera, K. Y. S., Priyanga Ranasinghe, A. M. M. C. Adikari, B. Balagobi, G. R. Constantine, and Saroj Jayasinghe. 2012. "Medium of Language in Discharge Summaries: Would the Use of Native Language Improve Patients' Knowledge of Their Illness and Medications?” Journal of Health Communication 17( 2): 141-48. doi:10.1080/10810730.2011.585926.

Plumbaum, Till, Sascha Narr, Elif Eryilmaz, Frank Hopfgartner, Funda Klein-Ellinghaus, Anna Reese, and Sahin Albayrak. 2014. "Providing Multilingual Access to Health-Related Content." Studies in Health Technology and Informatics 205: 393-97.

Prakash, Sateesh Reddy, Siddharth Verma, John McGowan, Betsy E. Smith, Anjali Shroff, Gregory H. Gibson, Michael Cheng, Douglas Lowe Ii, Kavitha Gopal, and Smruti R. Mohanty. 2013. "Improving the Quality of Colonoscopy Bowel Preparation Using an Educational Video." 
Canadian Journal of Gastroenterology = Journal Canadien De Gastroenterologie 27(12): 696700.

Sentell, Tetine, and Kathryn L. Braun. 2012. "Low Health Literacy, Limited English Proficiency, and Health Status in Asians, Latinos, and Other Racial/Ethnic Groups in California." Journal of Health Communication 17(Suppl 3): 82-99. doi:10.1080/10810730.2012.712621.

Statistics Canada. 2012. "2011 Census Profile.” Accessed April 5, 2017. http://www12.statcan.gc.ca/census-recensement/2011/dp-pd/prof/index.cfm?Lang=E.

Weiss, Linda, Francesca Gany, Peri Rosenfeld, Olveen Carrasquillo, Iman Sharif, Elana Behar, Emily Ambizas, Priti Patel, Lauren Schwartz, and Robert Mangione. 2007. "Access to Multilingual Medication Instructions at New York City Pharmacies." Journal of Urban Health: Bulletin of the New York Academy of Medicine 84( 6): 742-54. doi:10.1007/s11524-007-9221-3. Workman, T. Elizabeth, Nancy T. Lombardo, and Jeanne Marie Le Ber. 2003. "Overcoming Language Barriers When Providing Health Information." Journal of Consumer Health on the Internet 7(2): 23-33. doi:10.1300/J381v07n02_03. 


\section{Consumer \& Patient Health Information Service Diabetes Resource Guide (Multilingual)}

The University of Manitoba Health Sciences Libraries' Consumer Health Information Service provides both print and electronic resources for patients, families, and members of the general public. We have compiled online resource lists for patients who speak languages other than English. This topic includes the following languages:

- Amharic

- Arabic

- Bengali

- Bosnian

- Chinese

- Cree

- Farsi (Persian)

- German

- Greek

- Gujarati

- Hindi

- Italian

- Korean

- Lao (Laotian)

To see other available resource lists, or to request other topics or languages, please contact us: http://libguides.lib.umanitoba.ca/sogh
- Nepali

- Ojibwe

- Polish

- Portuguese

- Punjabi

- Russian

- Somalí

- Spanish

- Tagalog

- Ukrainian

- Urdu

- Vietnamese 


\section{Seven Oaks General Hospital Library \\ $204-632-3124$ - soghlibrary tumanitoba ca hitp:/hibguides lib umanitoba ca/sogh

\section{Consumer \& Patient Health Information Service Diabetes Guide (Cree)}

These sites give information in Cree on topics related to diabetes.

- Intro to diabetes: http://bit.ly/2gin3TR

- Just the Basics: http://bit.ly/2gPPLyM

- Just the Basics (video): http://bit.ly/2gPOWWF

- Type 2 diabetes: hittp://bit.ly/2gWtirR

- Diabetes and nutrition: btto://bit.ly/2giabid

- Exercise and diabetes: http://bit.ly/2ginwou

- Bodyweight and diabetes: http://bit.ly/2fQjvvT

- Alcohol and diabetes: http://bit.ly/2git5DW

- Diabetic foot care: http://bit.ly/2fQaa7h

- Monitoring glucose levels: http://bit.ly/2gWsyrg 